Annals of Pure and Applied Mathematics

Vol.19, No.2, 2019, 207-217

ISSN: 2279-087X (P), 2279-0888(online)

Published on 24 May 2019

Annals of

www.researchmathsci.org

DOI: http://dx.doi.org/10.22457/apam.613v19n2a10

Pure and Applied

Mathematics

\title{
Seventh and Eighth Order Triangular Sum Labeling of Graphs
}

\author{
C. Jayasekaran ${ }^{1}$ and C. Sunitha ${ }^{2}$ \\ ${ }^{1}$ Department of Mathematics, Pioneer Kumaraswamy College \\ Nagercoil-629003 Tamilnadu, India. \\ ${ }^{2}$ Department of Mathematics, Women's Christian College, Nagercoil-629001 \\ Tamilnadu, India. Email: sunithadavidjones@gmail.com \\ ${ }^{1}$ Corresponding author. Email: jaya_pkc@yahoo.com
}

Received 19 March 2019; accepted 14 May 2019

Abstract. A (p, q) graph $\mathrm{G}$ is said to admit a seventh (or eighth) order triangular sum labeling if its vertices can be labeled by non negative integers such that induced edge labels obtained by the sum of the labels of end vertices are the seventh (or eighth) order triangular numbers. A graph $\mathrm{G}$ which admits a seventh (or eighth) order triangular sum labeling is called a seventh (or eighth) order triangular sum graph. In this paper we prove that double star $\mathrm{K}_{1, \mathrm{n}, \mathrm{n}}, \mathrm{Tg}_{\mathrm{n}}$ and Coconut tree admit seventh and eighth order triangular sum labelings.

Keywords: Star graph, coconut tree, seventh and eighth order triangular sum graph

AMS Mathematics Subject Classification (2010): 05 C78

\section{Introduction}

The graphs considered here are finite, connected, undirected and simple. The vertex set and the edge set of graph are denoted by $\mathrm{V}(\mathrm{G})$ and $\mathrm{E}(\mathrm{G})$ respectively. For various graph theoretic notations and terminology we follow Harary [3] and for number theory we follow Burton [1]. We will give the brief summary of definitions which are useful for the present investigations. In [8] they have proved star and bistar related graphs are divisor cordial graphs. Some authors in [10] have discussed Star in Coloring of some star familes. For a dynamic survey of various graph labeling problem along with an extensive bibliography we refer to Gallioan [4].

Definition 1.1. [2,7 ] A triangular number is a number obtained by adding all positive integers less than or equal to a given positive integer $n$. If the $n^{\text {th }}$ triangular number is denoted by $A_{n}$, then $A_{n}=1+2+\ldots+n=\frac{1}{2} n(n+1)$.

The triangular numbers are $1,3,6,10,15,21,28,36, \ldots$

Definition 1.2. [6, 9] A triangular sum labeling of a graph $G$ is a one to one function $f$ : $\mathrm{V}(\mathrm{G}) \rightarrow \mathrm{W}$ (where $\mathrm{W}$ is the set of all non-negative integers) that induces a bijection $\mathrm{f}^{+}$: 


\section{Jayasekaran and C. Sunitha}

$E(G) \rightarrow\left\{A_{1}, A_{2}, \ldots ., A_{q}\right\}$ of the edges of $G$ defined by $f^{+}(u v)=f(u)+f(v)$ $\forall \mathrm{e}=\mathrm{uv} \in \mathrm{E}(\mathrm{G})$. The graph which admits such labeling is called a triangular sum graph.

Definition 1.3. [5] A second order triangular number is a number obtained by adding all the squares of positive integer less than or equal to a given positive integer $n$. If the $n^{\text {th }}$ second order triangular number is denoted by $B_{n}$, then

$$
\begin{aligned}
& \mathrm{B}_{\mathrm{n}}=1^{2}+2^{2}+3^{2}+\ldots+\mathrm{n}^{2} \\
& =\frac{1}{6} n(n+1)(2 n+1)
\end{aligned}
$$

The second order triangular numbers are $1,5,14,30,55, \ldots$

Definition 1.4. A second order triangular sum labeling of a graph $G$ is a one to one function $\mathrm{f:} V(\mathrm{G}) \rightarrow \mathrm{W}$ that induces a bijection $\mathrm{f}^{+}: \mathrm{E}(\mathrm{G}) \rightarrow\left\{\mathrm{B}_{1}, \mathrm{~B}_{2}, \ldots, \mathrm{B}_{\mathrm{n}}\right\}$ of the edges of $\mathrm{G}$ defined by $\mathrm{f}^{+}(\mathrm{uv})=\mathrm{f}(\mathrm{u})+\mathrm{f}(\mathrm{v}), \forall \mathrm{e}=\mathrm{uv} \in \mathrm{E}(\mathrm{G})$. The graph which admits such labeling is called a second order triangular sum graph.

Definition 1.5. A seventh order triangular number is a number obtained by adding all the seventh powers of positive integers less than or equal to a given positive integer $\mathrm{n}$. If the $\mathrm{n}^{\text {th }}$ seventh order triangular number is denoted by $\mathrm{G}_{\mathrm{n}}$, then

$$
\begin{aligned}
& \mathrm{G}_{\mathrm{n}}=1^{7}+2^{7}+3^{7}+\ldots+\mathrm{n}^{7} \\
& =\frac{1}{24} n^{2}(n+1)^{2}\left[3 n^{4}+6 n^{3}-n^{2}-4 n+2\right]
\end{aligned}
$$

The seventh order triangular numbers are 1, 129, 2316, 18700, 96825, 376761, $1200304,3297456,8080425,18080425,37567596, \ldots$

Definition 1.6. A seventh order triangular sum labeling of a graph $G$ is a one to one function $\mathrm{f}: \mathrm{V}(\mathrm{G}) \rightarrow \mathrm{W}$ that induces a bijection $\mathrm{f}^{+}: \mathrm{E}(\mathrm{G}) \rightarrow\left\{\mathrm{G}_{1}, \mathrm{G}_{2}, \ldots, \mathrm{G}_{\mathrm{q}}\right\}$ of the edges of $\mathrm{G}$ defined by $\mathrm{f}^{+}(\mathrm{uv})=\mathrm{f}(\mathrm{u})+\mathrm{f}(\mathrm{v}), \forall \mathrm{e}=\mathrm{uv} \in \mathrm{E}(\mathrm{G})$. The graph which admits such labeling is called a seventh order triangular sum graph.

Definition 1.7. An Eighth order triangular number is a number obtained by adding all the eighth powers of positive integers less than or equal to a given positive integer $n$. If the $\mathrm{n}^{\text {th }}$ eighth order triangular number is denoted by $\mathrm{H}_{\mathrm{n}}$, then

$$
\begin{aligned}
& \mathrm{H}_{\mathrm{n}}=1^{8}+2^{8}+\ldots+\mathrm{n}^{8} \\
& =\frac{n(n+1)(2 n+1)}{90}\left[5 n^{6}+15 n^{5}+5 n^{4}-15 n^{3}-n^{2}+9 n-3\right]
\end{aligned}
$$

The eighth order triangular numbers are $1,257,6818,72354,462979,2142595, \ldots$.

Definition 1.6. An Eighth order triangular sum labeling of a graph $G$ is a one-to-one function $\mathrm{f:} V(\mathrm{G}) \rightarrow \mathrm{W}$ that induces a bijection $\mathrm{f}^{+}: \mathrm{E}(\mathrm{G}) \rightarrow\left\{\mathrm{H}_{1}, \mathrm{H}_{2}, \ldots, \mathrm{H}_{\mathrm{q}}\right\}$ of the edges of $\mathrm{G}$ defined by $\mathrm{f}^{+}(\mathrm{uv})=\mathrm{f}(\mathrm{u})+\mathrm{f}(\mathrm{v}), \forall \mathrm{e}=\mathrm{uv} \in \mathrm{E}(\mathrm{G})$. The graph which admits such labeling is called a eighth order triangular sum graph. 


\section{Main result}

Theorem 2.1. The star graph $\mathrm{K}_{1, \mathrm{n}}$ admits seventh order triangular sum labeling.

Proof: Let $\mathrm{u}$ be the central vertex and let $\mathrm{u}_{1}, \mathrm{u}_{2,}, \ldots, \mathrm{u}_{\mathrm{n}}$ be the pendant vertices of the star $\mathrm{K}_{1, \mathrm{n}}$. Then the vertex set $\mathrm{V}\left(\mathrm{K}_{1, \mathrm{n}}\right)=\left\{\mathrm{u}, \mathrm{u}_{\mathrm{i}} / 1 \leq \mathrm{i} \leq \mathrm{n}\right\}$ and the edge set $\mathrm{E}\left(\mathrm{K}_{1, \mathrm{n}}\right)=\left\{\mathrm{uu}_{\mathrm{i}} / 1 \leq \mathrm{i}\right.$ $\leq \mathrm{n}\}$. Clearly $\mathrm{K}_{1, \mathrm{n}}$ has $\mathrm{n}+1$ vertices and $\mathrm{n}$ edges. Define $\mathrm{f}: \mathrm{V}\left(\mathrm{K}_{1, \mathrm{n}}\right) \rightarrow \mathrm{W}$ by

$\mathrm{f}(\mathrm{u})=0$

$\mathrm{f}\left(\mathrm{u}_{\mathrm{i}}\right)=\mathrm{G}_{\mathrm{i}}=\frac{i^{2}(i+1)^{2}}{24}\left[3 i^{4}+6 i^{3}-i^{2}-4 i+2\right], 1 \leq i \leq n$.

Then $\mathrm{f}$ induces a bijection $\mathrm{f}^{+}: \mathrm{E}(\mathrm{G}) \rightarrow\left\{\mathrm{G}_{1}, \mathrm{G}_{2}, \ldots, \mathrm{G}_{\mathrm{n}}\right\}$ given by

$\mathrm{f}^{+}\left(\mathrm{uu}_{\mathrm{i}}\right)=\mathrm{f}(\mathrm{u})+\mathrm{f}\left(\mathrm{u}_{\mathrm{i}}\right)=0+\mathrm{G}_{\mathrm{i}}=\mathrm{G}_{\mathrm{i}}=\frac{i^{2}(i+1)^{2}}{24}\left[3 i^{4}+6 i^{3}-i^{2}-4 i+2\right], 1 \leq \mathrm{i} \leq \mathrm{n}$.

Clearly, the induced edge labels are the first $\mathrm{n}$ seventh order triangular numbers. Hence $\mathrm{K}_{1, \mathrm{n}}$ admits a seventh order triangular sum labeling.

Example 2.2. A seventh order triangular sum labeling of $\mathrm{K}_{1,5}$ is shown in figure 2.1.

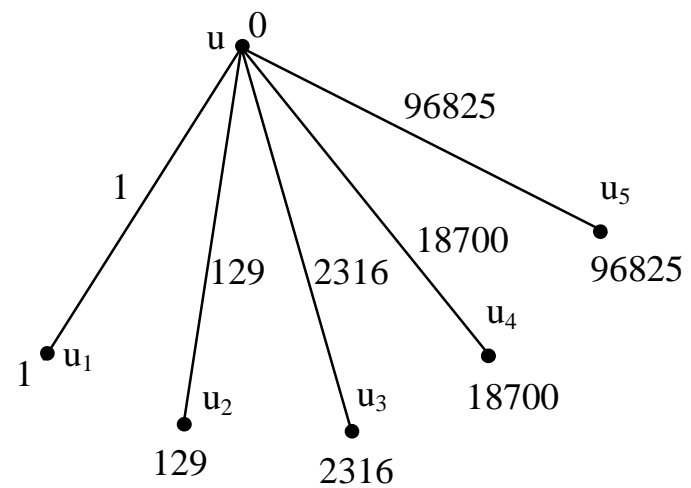

Figure 2.1: $\mathrm{K}_{1,5}$ with a seventh order triangular sum labeling

Theorem 2.3. The double star $\mathrm{K}_{1, \mathrm{n}, \mathrm{n}}$ admits seventh order triangular sum labeling.

Proof: Let $\mathrm{G}$ be the double star $\mathrm{K}_{1, \mathrm{n}, \mathrm{n}}$. Let $\mathrm{V}(\mathrm{G})=\left\{\mathrm{u}, \mathrm{u}_{\mathrm{i}}, \mathrm{v}_{\mathrm{i}} / 1 \leq \mathrm{i} \leq \mathrm{n}\right\}$ be the vertex set and $\mathrm{E}(\mathrm{G})=\left\{\mathrm{uu}_{\mathrm{i}}, \mathrm{u}_{\mathrm{i}} \mathrm{v}_{\mathrm{i}} / 1 \leq \mathrm{i} \leq \mathrm{n}\right\}$ be the edge set of $\mathrm{K}_{1, \mathrm{n}, \mathrm{n}}$. Then $\mathrm{G}$ has $2 \mathrm{n}+1$ vertices and $2 \mathrm{n}$ edges. Define $\mathrm{f}: \mathrm{V}(\mathrm{G}) \rightarrow \mathrm{W}$ by

$\mathrm{f}(\mathrm{u})=0$

$\mathrm{f}\left(\mathrm{u}_{\mathrm{i}}\right)=\mathrm{G}_{\mathrm{i}}, 1 \leq \mathrm{i} \leq \mathrm{n}$

$\mathrm{f}\left(\mathrm{v}_{\mathrm{i}}\right)=\mathrm{G}_{\mathrm{n}+\mathrm{i}}-\mathrm{f}\left(\mathrm{u}_{\mathrm{i}}\right), 1 \leq \mathrm{i} \leq \mathrm{n}$

Then $\mathrm{f}$ induces a bijection $\mathrm{f}^{+}: \mathrm{E}(\mathrm{G}) \rightarrow\left\{\mathrm{G}_{1}, \mathrm{G}_{2}, \ldots, \mathrm{G}_{2 \mathrm{n}}\right\}$ given by

$\mathrm{f}^{+}\left(\mathrm{uu}_{\mathrm{i}}\right)=\mathrm{f}(\mathrm{u})+\mathrm{f}\left(\mathrm{u}_{\mathrm{i}}\right)=0+\mathrm{G}_{\mathrm{i}}=\mathrm{G}_{\mathrm{i}}, 1 \leq \mathrm{i} \leq \mathrm{n}$

$\mathrm{f}^{+}\left(\mathrm{u}_{\mathrm{i}} \mathrm{v}_{\mathrm{i}}\right)=\mathrm{f}\left(\mathrm{u}_{\mathrm{i}}\right)+\mathrm{f}\left(\mathrm{v}_{\mathrm{i}}\right)=\mathrm{f}\left(\mathrm{u}_{\mathrm{i}}\right)+\mathrm{G}_{\mathrm{n}+\mathrm{I}}-\mathrm{f}\left(\mathrm{u}_{\mathrm{i}}\right)=\mathrm{G}_{\mathrm{n}+\mathrm{i}}, 1 \leq \mathrm{i} \leq \mathrm{n}$.

Thus, the induced edge labels are the first $2 n$ seventh order triangular numbers. Hence $G$ admits a seventh order triangular sum labeling.

Example 2.4. A seventh order triangular sum labeling of $\mathrm{K}_{1,4,4}$ is shown in figure 2.2. 


\section{Jayasekaran and C. Sunitha}

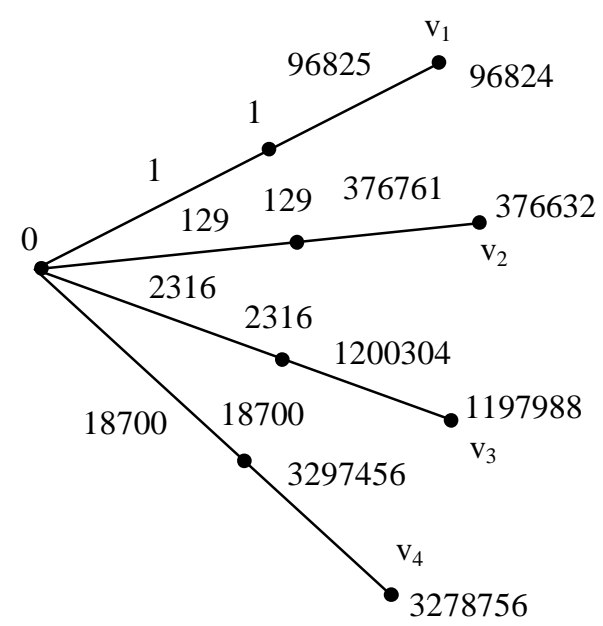

Figure 2.2: $\mathrm{K}_{1,4,4}$ with a seventh order triangular sum labeling

Theorem 2.5. The bistar $B_{m, n}$ admits seventh order triangular sum labeling.

Proof: Let $u$ and $v$ be the vertices of $K_{2}$. Join $m$ pendent vertices $u_{1}, u_{2}, \ldots, u_{m}$ at $u$ and join $n$ pendent vertices $\mathrm{v}_{1}, \mathrm{v}_{2}, \ldots, \mathrm{v}_{\mathrm{n}}$ at $\mathrm{v}$. The resultant graph is the bistar $\mathrm{G}=\mathrm{B}_{\mathrm{m}, \mathrm{n}}$ with vertex set $\mathrm{V}(\mathrm{G})=\left\{\mathrm{u}, \mathrm{v}, \mathrm{u}_{\mathrm{i}}, \mathrm{v}_{\mathrm{j}} / 1 \leq \mathrm{i} \leq \mathrm{m}, 1 \leq \mathrm{j} \leq \mathrm{n}\right\}$ and edge set $\mathrm{E}(\mathrm{G})\left\{\mathrm{uu}_{\mathrm{i}}, \mathrm{vv}_{\mathrm{j}}, \mathrm{uv} / 1\right.$ $\leq \mathrm{i} \leq \mathrm{m}, 1 \leq \mathrm{j} \leq \mathrm{n}\}$. Clearly $\mathrm{G}$ has $\mathrm{m}+\mathrm{n}+2$ vertices and $\mathrm{m}+\mathrm{n}+1$ edges. Define $\mathrm{f}: \mathrm{V}(\mathrm{G})$ $\rightarrow \mathrm{W}$ by

$\mathrm{f}(\mathrm{u})=0$

$\mathrm{f}(\mathrm{v})=1$

$\mathrm{f}\left(\mathrm{u}_{\mathrm{i}}\right)=\mathrm{G}_{\mathrm{i}+1}, \quad 1 \leq \mathrm{i} \leq \mathrm{m}$

$\mathrm{f}\left(\mathrm{v}_{\mathrm{j}}\right)=\mathrm{G}_{\mathrm{m}+\mathrm{1}+\mathrm{j}}-\mathrm{f}(\mathrm{v}), 1 \leq \mathrm{j} \leq \mathrm{n}$

Then $\mathrm{f}$ induces a bijection $\mathrm{f}^{+}: \mathrm{E}(\mathrm{G}) \rightarrow\left\{\mathrm{G}_{1}, \mathrm{G}_{2}, \ldots, \mathrm{G}_{\mathrm{m}+\mathrm{n}+1}\right)$ given by

$\mathrm{f}^{+}\left(\mathrm{uu}_{\mathrm{i}}\right)=\mathrm{f}(\mathrm{u})+\mathrm{f}\left(\mathrm{u}_{\mathrm{i}}\right)=0+\mathrm{G}_{\mathrm{i}+1}=\mathrm{G}_{\mathrm{i}+1}, \quad 1 \leq i \leq m$

$\mathrm{f}^{+}(\mathrm{uv})=\mathrm{f}(\mathrm{u})+\mathrm{f}(\mathrm{v})=1=\mathrm{G}_{1}$

$\mathrm{f}^{+}\left(\mathrm{vv}_{\mathrm{j}}\right)=\mathrm{f}(\mathrm{v})+\mathrm{f}\left(\mathrm{v}_{\mathrm{j}}\right)=\mathrm{f}(\mathrm{v})+\mathrm{G}_{\mathrm{m}+1+\mathrm{j}}-\mathrm{f}(\mathrm{v})=\mathrm{G}_{\mathrm{m}+1+\mathrm{j}}, 1 \leq \mathrm{j} \leq \mathrm{n}$.

Thus, the induced edge labels are the first $m+n+1$ seventh order triangular numbers. Hence $B_{m, n}$ admits a seventh order triangular sum labeling.

Example 2.6. A seventh order triangular sum labeling of $B_{4,3}$ is shown in figure 2.3.

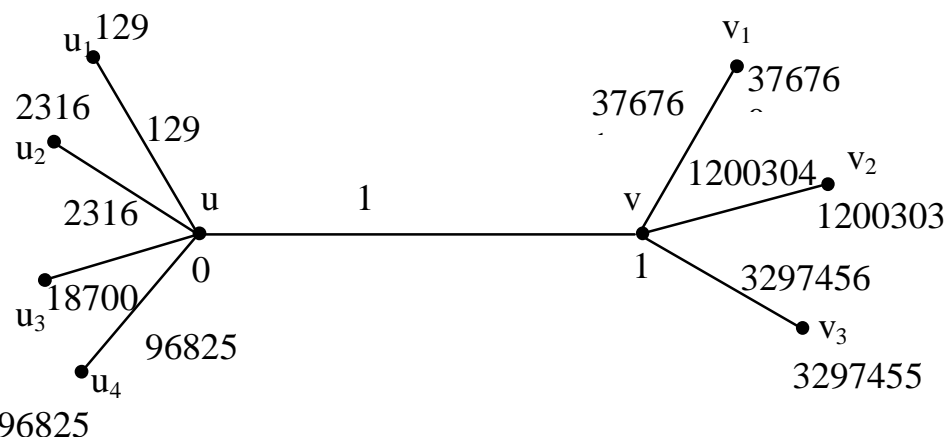


Seventh and Eighth Order Triangular Sum Labeling of Graphs

Figure 2.3: $\mathrm{B}_{4,3}$ with a seventh order triangular sum labeling

Theorem 2.7. $Y_{n+1}$ admits a seventh order triangular sum labeling if $n \geq 3$.

Proof: Let $P_{n}$ be the path $u_{1} u_{2}$. . . $u_{n}$. Let $v$ be the vertex adjacent to $u_{2}$. The resultant graph $G$ is $Y_{n+1}$ with vertex set $V(G)=\left\{u_{i}, v / 1 \leq i \leq n\right\}$ and the edge set $E(G)=\left\{u_{i} u_{i+1}\right.$, $\left.\mathrm{vu}_{2} / 1 \leq \mathrm{i} \leq \mathrm{n}-1\right\}$. Clearly the graph $\mathrm{G}$ has $\mathrm{n}+1$ vertices and $\mathrm{n}$ edges. Define $\mathrm{f}: \mathrm{V}(\mathrm{G}) \rightarrow$ W by

$\mathrm{f}\left(\mathrm{u}_{1}\right)=1=\mathrm{G}_{1}$

$\mathrm{f}(\mathrm{v})=129=\mathrm{G}_{2}$

$\mathrm{f}\left(\mathrm{u}_{2}\right)=0$

$\mathrm{f}\left(\mathrm{u}_{\mathrm{i}}\right)=\mathrm{G}_{\mathrm{i}+1}-\mathrm{f}\left(\mathrm{u}_{\mathrm{i}-1}\right), \quad 3 \leq \mathrm{i} \leq \mathrm{n}$

Then $\mathrm{f}$ induces a bijection $\mathrm{f}^{+}: \mathrm{E}(\mathrm{G}) \rightarrow\left\{\mathrm{G}_{1}, \mathrm{G}_{2}, \ldots, \mathrm{G}_{\mathrm{n}}\right\}$ given by

$\mathrm{f}^{+}\left(\mathrm{u}_{1} \mathrm{u}_{2}\right)=\mathrm{f}\left(\mathrm{u}_{1}\right)+\mathrm{f}\left(\mathrm{u}_{2}\right)=1=\mathrm{G}_{1}$

$\mathrm{f}^{+}\left(\mathrm{u}_{2} \mathrm{v}\right)=\mathrm{f}\left(\mathrm{u}_{2}\right)+\mathrm{f}(\mathrm{v})=0+129=\mathrm{G}_{2}$

$\mathrm{f}^{+}\left(\mathrm{u}_{2} \mathrm{u}_{3}\right)=\mathrm{f}\left(\mathrm{u}_{2}\right)+\mathrm{f}\left(\mathrm{u}_{3}\right)=0+23161=\mathrm{G}_{3}$

$\mathrm{f}^{+}\left(\mathrm{u}_{\mathrm{i}} \mathrm{u}_{\mathrm{i}+1}\right)=\mathrm{f}\left(\mathrm{u}_{\mathrm{i}}\right)+\mathrm{f}\left(\mathrm{u}_{\mathrm{i}+1}\right)=\mathrm{f}\left(\mathrm{u}_{\mathrm{i}}\right)+\mathrm{G}_{\mathrm{i}+1}-\mathrm{f}\left(\mathrm{u}_{\mathrm{i}}\right)=\mathrm{G}_{\mathrm{i}+1}, 3 \leq \mathrm{i} \leq \mathrm{n}$

Thus, the induced edge labels are the first $n$ seventh order triangular numbers. Hence $G$ admits seventh order triangular sum labeling.

Example 2.8. A seventh order triangular sum labeling of $Y_{5+1}$ is shown in figure 2.4.

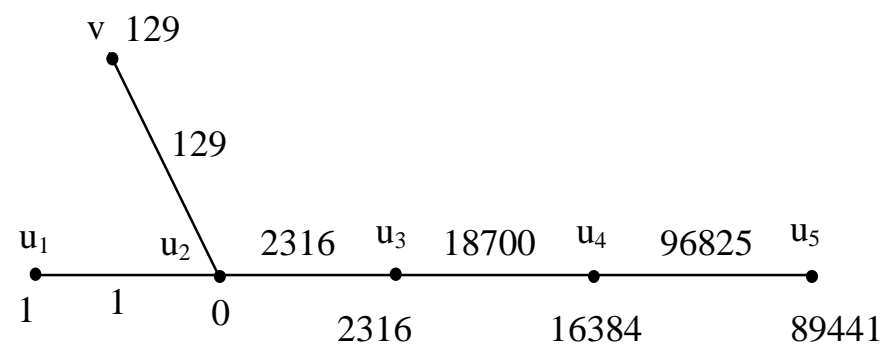

Figure 2.4: $\mathrm{Y}_{5+1}$ with a seventh order triangular sum labeling

Theorem 2.9. $\mathrm{Tg}_{\mathrm{n}}$ admits seventh order triangular sum labeling.

Proof: Let $u_{1} u_{2} \ldots u_{n}$ be the path of length $n$. For $1 \leq j \leq n-2$, let $v_{j}$ and $w_{j}$ be the vertices adjacent to $u_{j+1}$. The resultant graph is called a Twig graph $T g_{n}$, with vertex set $\mathrm{V}\left(\mathrm{Tg}_{\mathrm{n}}\right)=\left\{\mathrm{u}_{\mathrm{i}}, \mathrm{v}_{\mathrm{j}}, \mathrm{w}_{\mathrm{j}} / 1 \leq \mathrm{i} \leq \mathrm{n}, 1 \leq \mathrm{j} \leq \mathrm{n}-2\right\}$ and the edge set $\mathrm{E}\left(\mathrm{Tg}_{\mathrm{n}}\right)=\left\{\mathrm{u}_{\mathrm{i}} \mathrm{u}_{\mathrm{i}+1}, \mathrm{v}_{\mathrm{j}} \mathrm{u}_{\mathrm{j}+1}, \mathrm{w}_{\mathrm{j}} \mathrm{u}_{\mathrm{j}+1}\right.$ $/ 1 \leq \mathrm{i} \leq \mathrm{n}-1,1 \leq \mathrm{j} \leq \mathrm{n}-2\}$. Clearly the graph $\operatorname{Tg}_{\mathrm{n}}$ has $3 \mathrm{n}-4$ vertices and $3 \mathrm{n}-5$ edges. Define $\mathrm{f:} \mathrm{V}(\mathrm{G}) \rightarrow \mathrm{W}$ by

$\mathrm{f}\left(\mathrm{u}_{2}\right)=0$

$\mathrm{f}\left(\mathrm{u}_{3}\right)=\mathrm{G}_{1}$

$\mathrm{f}\left(\mathrm{u}_{\mathrm{i}}\right)=\mathrm{G}_{\mathrm{i}-2}-\mathrm{f}\left(\mathrm{u}_{\mathrm{i}-1}\right), 4 \leq \mathrm{i} \leq \mathrm{n}$

$\mathrm{f}\left(\mathrm{u}_{1}\right)=\mathrm{G}_{3 \mathrm{n}-5}$

$\mathrm{f}\left(\mathrm{v}_{\mathrm{i}}\right)=\mathrm{G}_{\mathrm{n}-2+\mathrm{i}}-\mathrm{f}\left(\mathrm{u}_{\mathrm{i}+1}\right), 1 \leq \mathrm{i} \leq \mathrm{n}-2$

$\mathrm{f}\left(\mathrm{w}_{\mathrm{i}}\right)=\mathrm{G}_{3 \mathrm{n}-5-\mathrm{i}}-\mathrm{f}\left(\mathrm{u}_{\mathrm{i}+1}\right), 1 \leq \mathrm{i} \leq \mathrm{n}-2$.

Then $\mathrm{f}$ induces a bijection $\mathrm{f}^{+}: \mathrm{E}(\mathrm{G}) \rightarrow\left\{\mathrm{G}_{1}, \mathrm{G}_{2}, \ldots, \mathrm{G}_{3 \mathrm{n}-5}\right\}$ given by

$\mathrm{f}^{+}\left(\mathrm{u}_{1} \mathrm{u}_{2}\right)=\mathrm{f}\left(\mathrm{u}_{1}\right)+\mathrm{f}\left(\mathrm{u}_{2}\right)=\mathrm{G}_{3 \mathrm{n}-5}$

$\mathrm{f}^{+}\left(\mathrm{u}_{\mathrm{i}} \mathrm{u}_{\mathrm{i}+1}\right)=\mathrm{f}\left(\mathrm{u}_{\mathrm{i}}\right)+\mathrm{f}\left(\mathrm{u}_{\mathrm{i}+1}\right)=\mathrm{G}_{\mathrm{i}-1}, 2 \leq \mathrm{i} \leq \mathrm{n}-1$

$\mathrm{f}^{+}\left(\mathrm{v}_{\mathrm{i}} \mathrm{u}_{\mathrm{i}+1}\right)=\mathrm{f}\left(\mathrm{v}_{\mathrm{i}}\right)+\mathrm{f}\left(\mathrm{u}_{\mathrm{i}+1}\right)=\mathrm{G}_{\mathrm{n}-2+\mathrm{i}}-\mathrm{f}\left(\mathrm{u}_{\mathrm{i}+1}\right)+\mathrm{f}\left(\mathrm{u}_{\mathrm{i}+1}\right)=\mathrm{G}_{\mathrm{n}-2+\mathrm{i}}, 1 \leq \mathrm{i} \leq \mathrm{n}-2$. 


\section{Jayasekaran and C. Sunitha}

$\mathrm{f}^{+}\left(\mathrm{w}_{\mathrm{i}} \mathrm{u}_{\mathrm{i}+1}\right)=\mathrm{f}\left(\mathrm{w}_{\mathrm{i}}\right)+\mathrm{f}\left(\mathrm{u}_{\mathrm{i}+1}\right)=\mathrm{G}_{3 \mathrm{n}-5-\mathrm{i}}+\mathrm{f}\left(\mathrm{w}_{\mathrm{i}}\right)-\mathrm{f}\left(\mathrm{w}_{\mathrm{i}}\right)=\mathrm{G}_{3 \mathrm{n}-5-\mathrm{i}}, \quad 1 \leq \mathrm{i} \leq \mathrm{n}-2$.

Thus, the induced edge labels are the first $3 n-5$ seventh order triangular numbers. Hence $\mathrm{Tg}_{\mathrm{n}}$ admits a seventh order triangular sum labeling.

Example 2.10. A seventh order triangular sum labeling of $\mathrm{Tg}_{5}$ is shown in figure 2.5

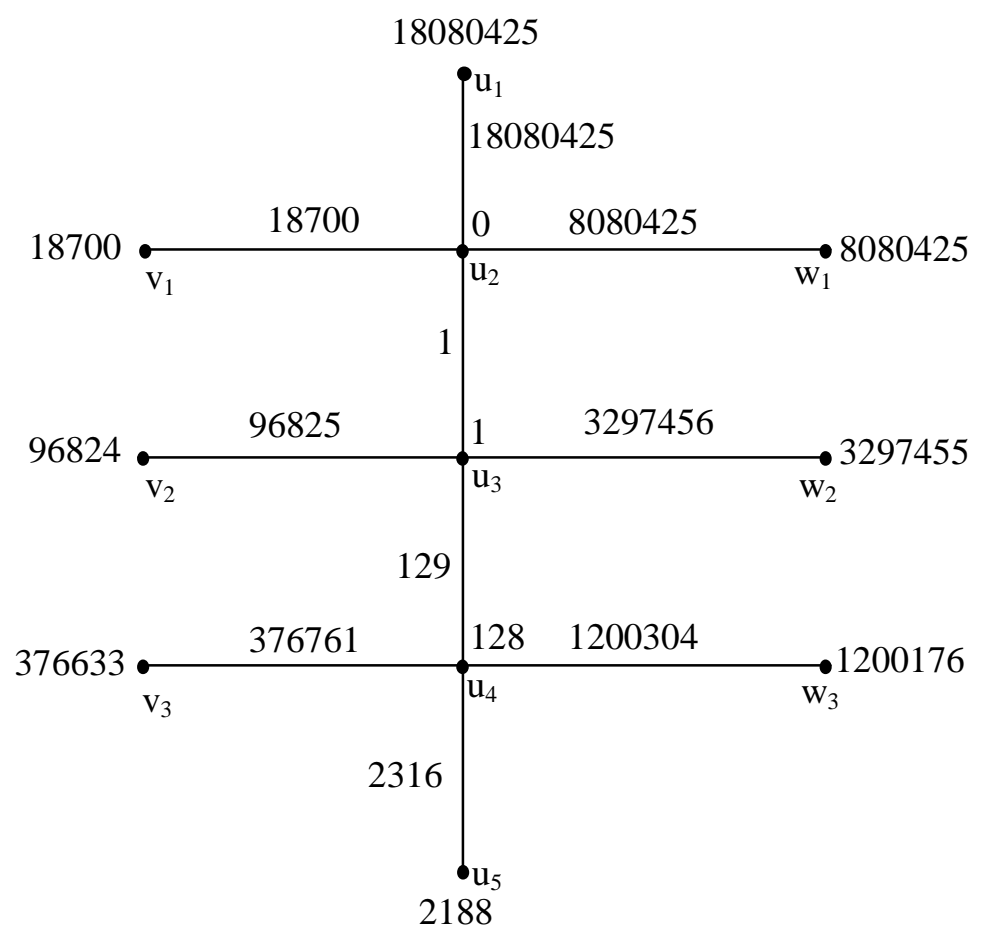

Figure 2.5: $\operatorname{Tg}_{5}$ with a seventh order triangular sum labeling

Theorem 2.11. The Coconut tree $\mathrm{T}(\mathrm{m}, \mathrm{n})$ admits a seventh order triangular sum labeling. Proof: Let $u_{1}, u_{2}, \ldots, u_{n}$ be the end vertices of star $K_{1, n}$ with central vertex $u$. Let $v_{1}, v_{2}$, $\ldots, \mathrm{v}_{\mathrm{m}}$ be the path $\mathrm{P}_{\mathrm{m}}$. Identifying $\mathrm{v}_{1}$ with $\mathrm{u}$. The resultant graph $\mathrm{G}$ is a coconut tree $\mathrm{T}(\mathrm{m}, \mathrm{n})$ with vertex set $\mathrm{V}(\mathrm{G})=\left\{\mathrm{v}_{\mathrm{i}}, \mathrm{u}_{\mathrm{j}} / 1 \leq \mathrm{i} \leq \mathrm{m}, 1 \leq \mathrm{j} \leq \mathrm{n}\right\}$ and edge set $\mathrm{E}(\mathrm{G})=\left\{\mathrm{v}_{\mathrm{i}} \mathrm{v}_{\mathrm{i}+1}, \mathrm{v}_{1} \mathrm{u}_{\mathrm{j}}, / 1 \leq \mathrm{j}\right.$ $\leq \mathrm{n}, 1 \leq \mathrm{i} \leq \mathrm{m}-1\}$. Clearly $\mathrm{G}$ has $\mathrm{n}+\mathrm{m}$ vertices and $\mathrm{n}+\mathrm{m}-1$ edges. Define $\mathrm{f}: \mathrm{V}(\mathrm{G}) \rightarrow$ W by

$\mathrm{f}\left(\mathrm{v}_{1}\right)=0$

$\mathrm{f}\left(\mathrm{v}_{\mathrm{i}}\right)=\mathrm{G}_{\mathrm{i}-1}-\mathrm{f}\left(\mathrm{v}_{\mathrm{i}-1}\right), 2 \leq \mathrm{i} \leq \mathrm{m}$

$\mathrm{f}\left(\mathrm{u}_{\mathrm{j}}\right)=\mathrm{G}_{\mathrm{m}+\mathrm{j}-1}, \quad 1 \leq \mathrm{j} \leq \mathrm{n}$.

Then $\mathrm{f}$ induces a bijection $\mathrm{f}^{+}: \mathrm{E}(\mathrm{G}) \rightarrow\left\{\mathrm{G}_{1}, \mathrm{G}_{2}, \ldots, \mathrm{G}_{\mathrm{m}+\mathrm{n}-1}\right\}$ given by

$\mathrm{f}^{+}\left(\mathrm{v}_{\mathrm{i}} \mathrm{v}_{\mathrm{i}+1}\right)=\mathrm{f}\left(\mathrm{v}_{\mathrm{i}}\right)+\mathrm{f}\left(\mathrm{v}_{\mathrm{i}+1}\right)=\mathrm{f}\left(\mathrm{v}_{\mathrm{i}}\right)+\mathrm{G}_{\mathrm{i}}-\mathrm{f}\left(\mathrm{v}_{\mathrm{i}}\right)=\mathrm{G}_{\mathrm{i}}, 1 \leq \mathrm{i} \leq \mathrm{m}-1$

$\mathrm{f}^{*}\left(\mathrm{v}_{1} \mathrm{u}_{\mathrm{j}}\right)=\mathrm{f}\left(\mathrm{v}_{1}\right)+\mathrm{f}\left(\mathrm{u}_{\mathrm{j}}\right)=0+\mathrm{G}_{\mathrm{m}+\mathrm{j}-1}=\mathrm{G}_{\mathrm{m}+\mathrm{j}-1}, 1 \leq \mathrm{j} \leq \mathrm{n}$.

Thus, the induced edge labels are the first $\mathrm{n}+\mathrm{m}-1$ seventh order triangular numbers.

Hence $\mathrm{G}$ admits a seventh order triangular sum labeling. 
Seventh and Eighth Order Triangular Sum Labeling of Graphs

Example 2.12. A seventh order triangular sum labeling of $T(4,5)$ is shown in figure 2.6.

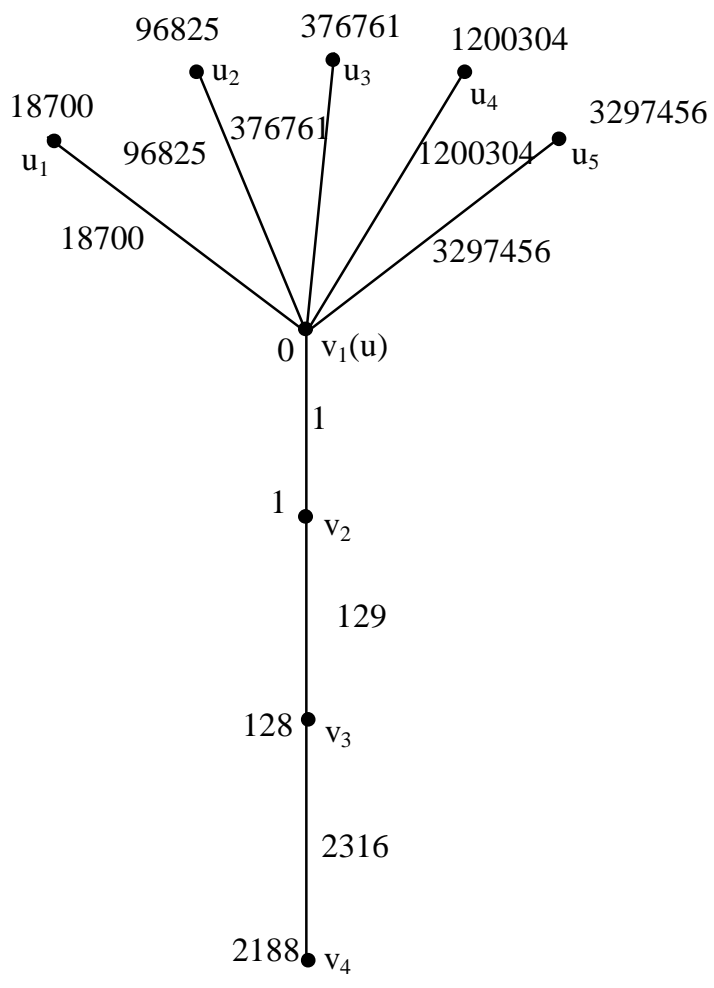

Figure 2.6: $\mathrm{T}(4,5)$ with a seventh order triangular sum labeling

Theorem 2.13. The star graph $\mathrm{K}_{1, \mathrm{n}}$ admits an eighth order triangular sum labeling.

Proof: Let $\mathrm{u}$ be the central vertex and let $\mathrm{u}_{1}, \mathrm{u}_{2,} \ldots, \mathrm{u}_{\mathrm{n}}$ be the pendant vertices of the star $\mathrm{K}_{1, \mathrm{n}}$. Then the vertex set $\mathrm{V}\left(\mathrm{K}_{1, \mathrm{n}}\right)=\left\{\mathrm{u}, \mathrm{u}_{\mathrm{i}} / 1 \leq \mathrm{i} \leq \mathrm{n}\right\}$ and the edge set $\mathrm{E}\left(\mathrm{K}_{1, \mathrm{n}}\right)=\left\{\mathrm{uu}_{\mathrm{i}} / 1 \leq\right.$ $\mathrm{i} \leq \mathrm{n}\}$. Clearly $\mathrm{K}_{1, \mathrm{n}}$ has $\mathrm{n}+1$ vertices and $\mathrm{n}$ edges. Define $\mathrm{f}: \mathrm{V}\left(\mathrm{K}_{1, \mathrm{n}}\right) \rightarrow \mathrm{W}$ by

$\mathrm{f}(\mathrm{u})=0$

$\mathrm{f}\left(\mathrm{u}_{\mathrm{i}}\right)=\mathrm{H}_{\mathrm{i}}, 1 \leq \mathrm{i} \leq \mathrm{n}$.

Then $\mathrm{f}$ induces a bijection $\mathrm{f}^{+}: \mathrm{E}(\mathrm{G}) \rightarrow\left\{\mathrm{H}_{1}, \mathrm{H}_{2}, \ldots, \mathrm{H}_{\mathrm{n}}\right\}$ given by $\mathrm{f}^{+}\left(\mathrm{uu}_{\mathrm{i}}\right)=\mathrm{f}(\mathrm{u})+\mathrm{f}\left(\mathrm{u}_{\mathrm{i}}\right)=0+\mathrm{H}_{\mathrm{i}}=\mathrm{H}_{\mathrm{i}}, \quad 1 \leq \mathrm{i} \leq \mathrm{n}$.

Cleary, the induced edge labels are the first $\mathrm{n}$ eighth order triangular numbers. Hence $\mathrm{K}_{1, \mathrm{n}}$ admits an eighth order triangular sum labeling.

Example 2.14. An eighth order triangular sum labeling of $\mathrm{K}_{1,5}$ is shown in Figure 2.7. 


\section{Jayasekaran and C. Sunitha}

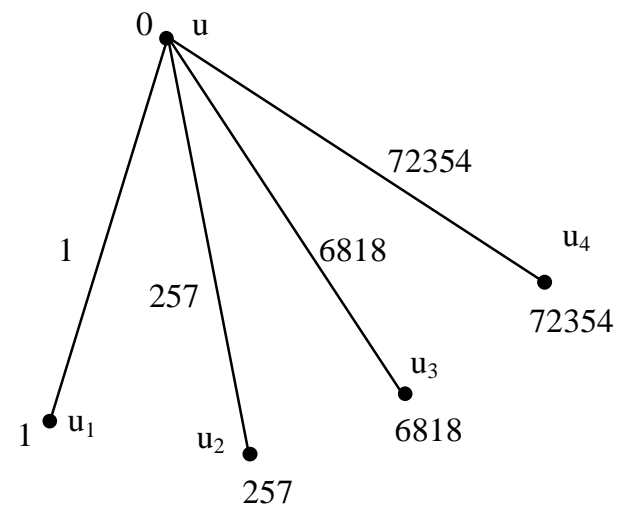

Figure 2.7: $\mathrm{K}_{1,4}$ with an eighth order triangular sum labeling

Theorem 2.15. The double star $\mathrm{K}_{1, \mathrm{n}, \mathrm{n}}$ admits an eighth order triangular sum labeling.

Proof: Let $\mathrm{G}$ be the double star $\mathrm{K}_{1, \mathrm{n}, \mathrm{n}}$. Let $\mathrm{V}(\mathrm{G})=\left\{\mathrm{u}, \mathrm{u}_{\mathrm{i}}, \mathrm{v}_{\mathrm{i}} / 1 \leq \mathrm{i} \leq \mathrm{n}\right\}$ be the vertex set and $E(G)=\left\{u_{i}, u_{i} v_{i} / 1 \leq \mathrm{i} \leq n\right\}$ be the edge set of $K_{1, n, n}$. Then $G$ has $2 n+1$ vertices and $2 \mathrm{n}$ edges. Define $\mathrm{f}: \mathrm{V}(\mathrm{G}) \rightarrow \mathrm{W}$ by

$\mathrm{f}(\mathrm{u})=0$

$\mathrm{f}\left(\mathrm{u}_{\mathrm{i}}\right)=\mathrm{H}_{\mathrm{i}}, \quad 1 \leq \mathrm{i} \leq \mathrm{n}$

$\mathrm{f}\left(\mathrm{v}_{\mathrm{i}}\right)=\mathrm{H}_{\mathrm{n}+\mathrm{i}}-\mathrm{f}\left(\mathrm{u}_{\mathrm{i}}\right), 1 \leq \mathrm{i} \leq \mathrm{n}$

Then $\mathrm{f}$ induces a bijection $\mathrm{f}^{+}: \mathrm{E}(\mathrm{G}) \rightarrow\left\{\mathrm{H}_{1}, \mathrm{H}_{2}, \ldots, \mathrm{H}_{2 \mathrm{n}}\right\}$ given by

$\mathrm{f}^{+}\left(\mathrm{uu}_{\mathrm{i}}\right)=\mathrm{f}(\mathrm{u})+\mathrm{f}\left(\mathrm{u}_{\mathrm{i}}\right)=0+\mathrm{H}_{\mathrm{i}}=\mathrm{H}_{\mathrm{i}}, 1 \leq \mathrm{i} \leq \mathrm{n}$

$\mathrm{f}^{+}\left(\mathrm{u}_{\mathrm{i}} \mathrm{v}_{\mathrm{i}}\right)=\mathrm{f}\left(\mathrm{u}_{\mathrm{i}}\right)+\mathrm{f}\left(\mathrm{v}_{\mathrm{i}}\right)=\mathrm{f}\left(\mathrm{u}_{\mathrm{i}}\right)+\mathrm{H}_{\mathrm{n}+\mathrm{I}}-\mathrm{f}\left(\mathrm{u}_{\mathrm{i}}\right)=\mathrm{H}_{\mathrm{n}+\mathrm{i}}, \quad 1 \leq \mathrm{i} \leq \mathrm{n}$.

Thus, the induced edge labels are the first $2 n$ eighth order triangular numbers. Hence $G$ admits an eighth order triangular sum labeling.

Example 2.16. An eighth order triangular sum labeling of double star $K_{1,3,3}$ is shown in figure 2.8.

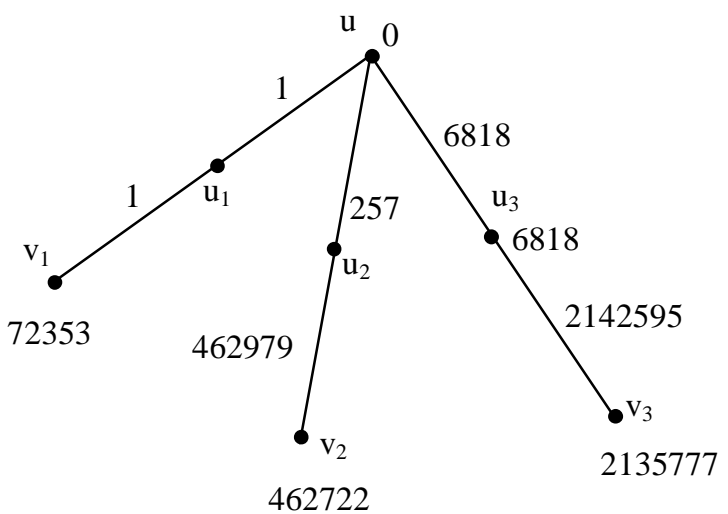

Figure 2.8: Double star $\mathrm{K}_{1,3,3}$ with an eighth order triangular sum labeling 
Seventh and Eighth Order Triangular Sum Labeling of Graphs

Theorem 2.17. $Y_{n+1}$ admits an eighth order triangular sum labeling.

Proof: Let $P_{n}$ be the path $u_{1} u_{2}$. . $u_{n}$. Let $v$ be the vertex adjacent to $u_{2}$. The resultant graph $G$ is $Y_{n+1}$ with vertex set $V(G)=\left\{u_{i}, v / 1 \leq i \leq n\right\}$ and the edge set $E(G)=\left\{u_{i} u_{i+1}\right.$, $\left.\mathrm{vu}_{2} / 1 \leq \mathrm{i} \leq \mathrm{n}-1\right\}$. Clearly the graph $\mathrm{G}$ has $\mathrm{n}+1$ vertices and $\mathrm{n}$ edges. Define $\mathrm{f}: \mathrm{V}(\mathrm{G}) \rightarrow$ W by

$\mathrm{f}\left(\mathrm{u}_{1}\right)=1$

$\mathrm{f}\left(\mathrm{u}_{2}\right)=0$

$\mathrm{f}(\mathrm{v})=257$

$\mathrm{f}\left(\mathrm{u}_{\mathrm{i}}\right)=\mathrm{H}_{\mathrm{i}}-\mathrm{f}\left(\mathrm{u}_{\mathrm{i}-1)}, 3 \leq \mathrm{i} \leq \mathrm{n}\right.$.

Then $\mathrm{f}$ induces a bijection $\mathrm{f}^{+}: \mathrm{E}(\mathrm{G}) \rightarrow\left\{\mathrm{H}_{1}, \mathrm{H}_{2}, \ldots, \mathrm{H}_{\mathrm{n}}\right\}$ given by

$\mathrm{f}^{+}\left(\mathrm{u}_{1} \mathrm{u}_{2}\right)=\mathrm{f}\left(\mathrm{u}_{1}\right)+\mathrm{f}\left(\mathrm{u}_{2}\right)=1=\mathrm{H}_{1}$

$\mathrm{f}^{+}\left(\mathrm{u}_{2} \mathrm{v}\right)=\mathrm{f}\left(\mathrm{u}_{2}\right)+\mathrm{f}(\mathrm{v})=257=\mathrm{H}_{2}$

$\mathrm{f}^{+}\left(\mathrm{u}_{2} \mathrm{u}_{3}\right)=\mathrm{f}\left(\mathrm{u}_{2}\right)+\mathrm{f}\left(\mathrm{u}_{3}\right)=0+6818=\mathrm{H}_{3}$

$\mathrm{f}^{+}\left(\mathrm{u}_{\mathrm{i}} \mathrm{u}_{\mathrm{i}+1}\right)=\mathrm{f}\left(\mathrm{u}_{\mathrm{i}}\right)+\mathrm{f}\left(\mathrm{u}_{\mathrm{i}+1}\right)=\mathrm{f}\left(\mathrm{u}_{\mathrm{i}}\right)+\mathrm{H}_{\mathrm{i}+1}-\mathrm{f}\left(\mathrm{u}_{\mathrm{i}}\right)=\mathrm{H}_{\mathrm{i}+1}, 3 \leq \mathrm{i} \leq \mathrm{n}$.

Cleary, the induced edge labels are the first $n$ eighth order triangular numbers. Hence $G$ admits an eighth order triangular sum labeling.

Example 2.18. An eighth order triangular sum labeling of $\mathbf{Y}_{5+1}$ is shown in figure 2.9.

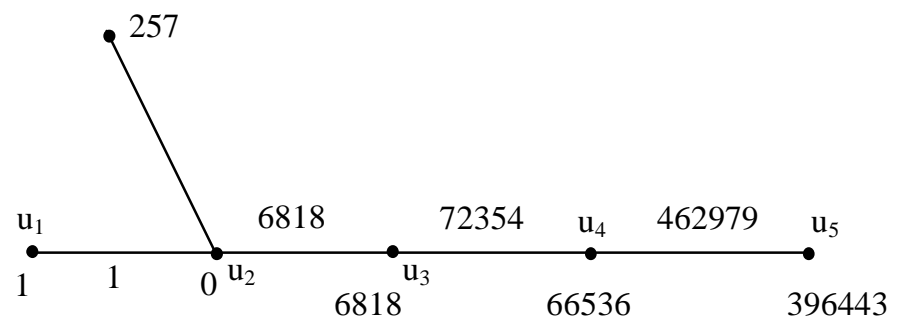

Figure 2.9: $Y_{5+1}$ with an eighth order triangular sum labeling

Theorem 2.19. $\mathrm{Tg}_{\mathrm{n}}$ admits an eighth order triangular sum labeling.

Proof: Let $u_{1} u_{2} \ldots u_{n}$ be the path of length $n$. For $1 \leq j \leq n-2$, let $v_{j}$ and $w_{j}$ be the vertices adjacent to $u_{j+1}$. The resultant graph is called a Twig graph $\operatorname{Tg}_{\mathrm{n}}$, with vertex set $\mathrm{V}\left(\mathrm{Tg}_{\mathrm{n}}\right)=\left\{\mathrm{u}_{\mathrm{i}}, \mathrm{v}_{\mathrm{j}}, \mathrm{w}_{\mathrm{j}} / 1 \leq \mathrm{i} \leq \mathrm{n}, 1 \leq \mathrm{j} \leq \mathrm{n}-2\right\}$ and the edge set $\mathrm{E}\left(\mathrm{Tg}_{\mathrm{n}}\right)=\left\{\mathrm{u}_{\mathrm{i}} \mathrm{u}_{\mathrm{i}+1}, \mathrm{v}_{\mathrm{j}} \mathrm{u}_{\mathrm{j}+1}, \mathrm{w}_{\mathrm{j}} \mathrm{u}_{\mathrm{j}+1}\right.$ $/ 1 \leq \mathrm{i} \leq \mathrm{n}-1,1 \leq \mathrm{j} \leq \mathrm{n}-2\}$. Clearly the graph $\mathrm{Tg}_{\mathrm{n}}$ has $3 \mathrm{n}-4$ vertices and $3 \mathrm{n}-5$ edges. Define $\mathrm{f:} \mathrm{V}(\mathrm{G}) \rightarrow \mathrm{W}$ by

$\mathrm{f}\left(\mathrm{u}_{2}\right)=0$

$\mathrm{f}\left(\mathrm{u}_{3}\right)=\mathrm{H}_{1}$

$\mathrm{f}\left(\mathrm{u}_{\mathrm{i}}\right)=\mathrm{H}_{\mathrm{i}-2}-\mathrm{f}\left(\mathrm{u}_{\mathrm{i}-1}\right), 4 \leq \mathrm{i} \leq \mathrm{n}$

$\mathrm{f}\left(\mathrm{u}_{1}\right)=\mathrm{H}_{3 \mathrm{n}-5}$

$\mathrm{f}\left(\mathrm{v}_{\mathrm{i}}\right)=\mathrm{H}_{\mathrm{n}-2+\mathrm{i}}-\mathrm{f}\left(\mathrm{u}_{\mathrm{i}+1}\right), 1 \leq \mathrm{i} \leq \mathrm{n}-2$

$\mathrm{f}\left(\mathrm{w}_{\mathrm{i}}\right)=\mathrm{H}_{3 \mathrm{n}-5-\mathrm{i}}-\mathrm{f}\left(\mathrm{u}_{\mathrm{i}+1}\right), 1 \leq \mathrm{i} \leq \mathrm{n}-2$.

Then $\mathrm{f}$ induces a bijection $\mathrm{f}^{+}: \mathrm{E}(\mathrm{G}) \rightarrow\left\{\mathrm{H}_{1}, \mathrm{H}_{2}, \ldots, \mathrm{H}_{3 \mathrm{n}-5}\right\}$ given by

$\mathrm{f}^{+}\left(\mathrm{u}_{1} \mathrm{u}_{2}\right)=\mathrm{f}\left(\mathrm{u}_{1}\right)+\mathrm{f}\left(\mathrm{u}_{2}\right)=\mathrm{H}_{3 \mathrm{n}-5}$

$\mathrm{f}^{+}\left(\mathrm{u}_{\mathrm{i}} \mathrm{u}_{\mathrm{i}+1}\right)=\mathrm{f}\left(\mathrm{u}_{\mathrm{i}}\right)+\mathrm{f}\left(\mathrm{u}_{\mathrm{i}+1}\right)=\mathrm{H}_{\mathrm{i}-1}, \quad 2 \leq \mathrm{i} \leq \mathrm{n}-1$

$\mathrm{f}^{+}\left(\mathrm{v}_{\mathrm{i}} \mathrm{u}_{\mathrm{i}+1}\right)=\mathrm{f}\left(\mathrm{v}_{\mathrm{i}}\right)+\mathrm{f}\left(\mathrm{u}_{\mathrm{i}+1}\right)=\mathrm{H}_{\mathrm{n}-2+\mathrm{i}}, \quad 1 \leq \mathrm{i} \leq \mathrm{n}-2$.

$\mathrm{f}^{+}\left(\mathrm{w}_{\mathrm{i}} \mathrm{u}_{\mathrm{i}+1}\right)=\mathrm{f}\left(\mathrm{w}_{\mathrm{i}}\right)+\mathrm{f}\left(\mathrm{u}_{\mathrm{i}+1}\right)=\mathrm{H}_{3 \mathrm{n}-5-\mathrm{i}}, 1 \leq \mathrm{i} \leq \mathrm{n}-2$. 


\section{Jayasekaran and C. Sunitha}

Cleary, the induced edge labels are the first $3 n-5$ eighth order triangular numbers. Hence $\mathrm{Tg}_{\mathrm{n}}$ admits an eighth order triangular sum labeling.

Example 2.20. An eighth order triangular sum labeling of $\mathrm{Tg}_{4}$ is shown in figure 2.10.

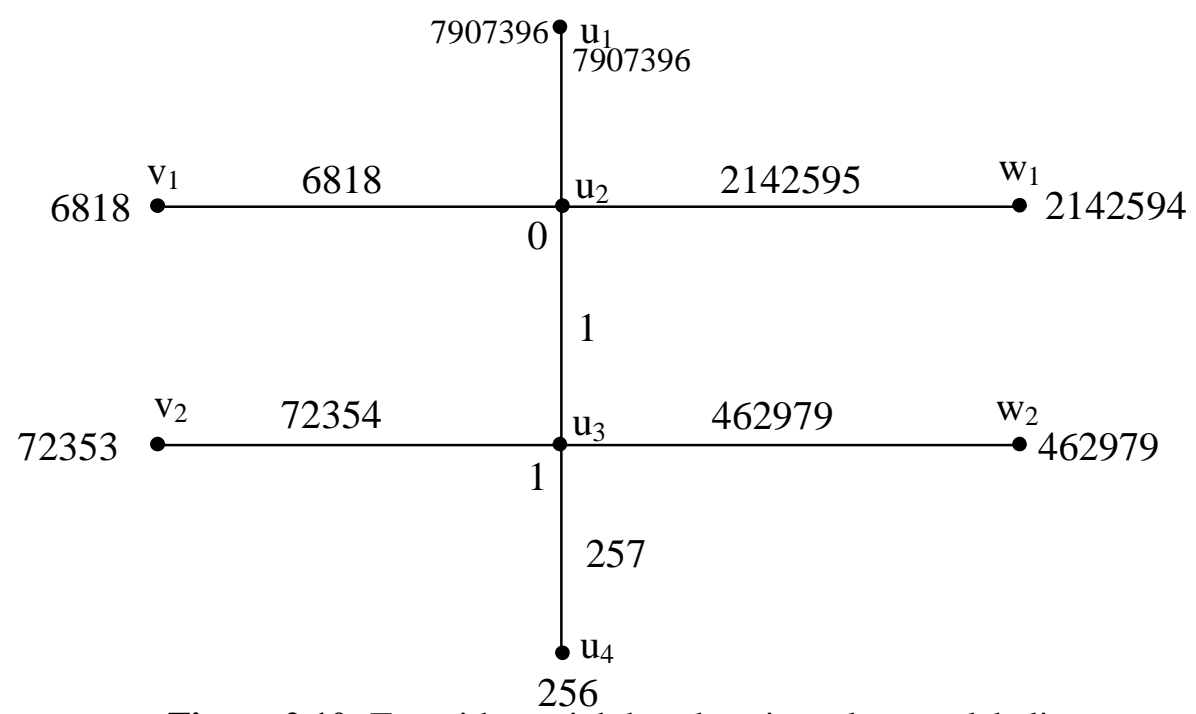

Figure 2.10: $\mathrm{Tg}_{4}$ with an eighth order triangular sum labeling

Theorem 2.21. The coconut tree $\mathrm{T}(\mathrm{m}, \mathrm{n})$ is an eighth order triangular sum labeling.

Proof: Let $\mathrm{u}_{1}, \mathrm{u}_{2}, \ldots, \mathrm{u}_{\mathrm{n}}$ be the end vertices of star $\mathrm{K}_{1, \mathrm{n}}$ with central vertex $\mathrm{u}$. Let $\mathrm{v}_{1}, \mathrm{v}_{2}, \ldots, \mathrm{v}_{\mathrm{m}}$ be the path $\mathrm{P}_{\mathrm{m}}$. Identifying $\mathrm{v}_{1}$ with $\mathrm{u}$. The resultant graph $\mathrm{G}$ is a coconut tree $\mathrm{T}(\mathrm{m}, \mathrm{n})$ with vertex set $\mathrm{V}(\mathrm{G})=\left\{\mathrm{v}_{\mathrm{i}}, \mathrm{u}_{\mathrm{j}} / 1 \leq \mathrm{i} \leq \mathrm{m}, 1 \leq \mathrm{j} \leq \mathrm{n}\right\}$ and edge set $\mathrm{E}(\mathrm{G})=\left\{\mathrm{v}_{\mathrm{i}} \mathrm{v}_{\mathrm{i}+1}\right.$, $\left.\mathrm{v}_{1} \mathrm{u}_{\mathrm{j},} / 1 \leq \mathrm{j} \leq \mathrm{n}, 1 \leq \mathrm{i} \leq \mathrm{m}-1\right\}$. Clearly $\mathrm{G}$ has $\mathrm{n}+\mathrm{m}$ vertices and $\mathrm{n}+\mathrm{m}-1$ edges. Define $\mathrm{f}$ : $\mathrm{V}(\mathrm{G}) \rightarrow \mathrm{W}$ by

$\mathrm{f}\left(\mathrm{v}_{1}\right)=0$

$\mathrm{f}\left(\mathrm{v}_{\mathrm{i}}\right)=\mathrm{H}_{\mathrm{i}-1}-\mathrm{f}\left(\mathrm{v}_{\mathrm{i}-1}\right), \quad 2 \leq \mathrm{i} \leq \mathrm{m}$

$\mathrm{f}\left(\mathrm{u}_{\mathrm{j}}\right)=\mathrm{H}_{\mathrm{n}+\mathrm{j}-1}, \quad 1 \leq \mathrm{i} \leq \mathrm{n}$.

Then $\mathrm{f}$ induces a bijection $\mathrm{f}^{+}: \mathrm{E}(\mathrm{G}) \rightarrow\left\{\mathrm{H}_{1}, \mathrm{H}_{2}, \ldots, \mathrm{H}_{\mathrm{m}+\mathrm{n}-1}\right\}$ given by

$\mathrm{f}^{+}\left(\mathrm{v}_{\mathrm{i}} \mathrm{v}_{\mathrm{i}+1}\right)=\mathrm{f}\left(\mathrm{v}_{\mathrm{i}}\right)+\mathrm{f}\left(\mathrm{v}_{\mathrm{i}+1}\right)=\mathrm{H}_{\mathrm{i}}, 1 \leq \mathrm{i} \leq \mathrm{m}-1$

$\mathrm{f}^{*}\left(\mathrm{v}_{1} \mathrm{u}_{\mathrm{j}}\right)=\mathrm{f}\left(\mathrm{v}_{1}\right)+\mathrm{f}\left(\mathrm{u}_{\mathrm{j}}\right)=\mathrm{H}_{\mathrm{m}+\mathrm{j}-1}, 1 \leq \mathrm{j} \leq \mathrm{n}$.

Cleary, the induced edge labels are the first $n+m-1$ eighth order triangular numbers. Hence $\mathrm{G}$ admits an eighth order triangular sum labeling.

Example 2.22. An eighth order triangular sum labeling of $T(4,4)$ is shown in Figure 2.11 . 
Seventh and Eighth Order Triangular Sum Labeling of Graphs

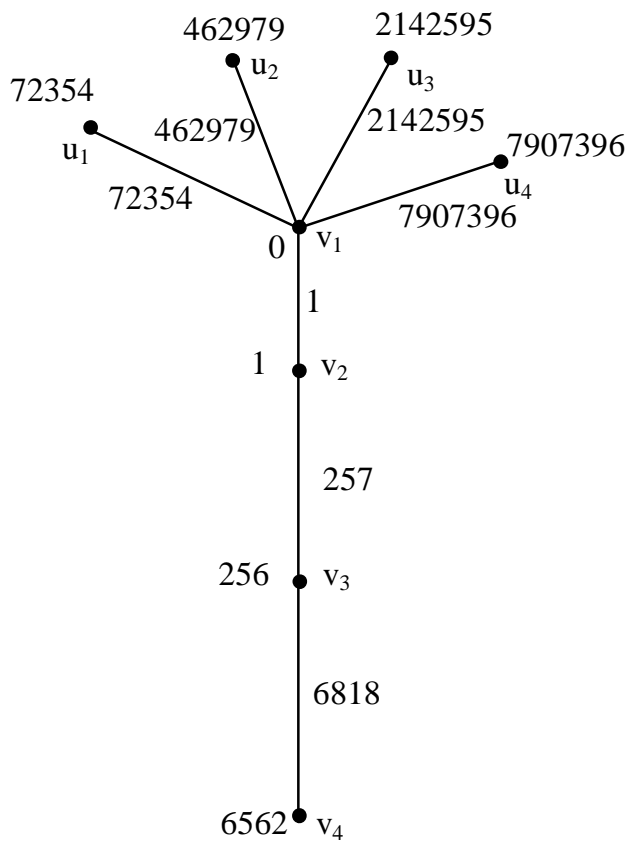

Figure 2.11: Coconut tree $\mathrm{T}(4,4)$ with an eighth order triangular sum labeling

\section{Conclusion}

In this paper, we prove the seventh and eighth order triangular sum labeling of star graph $\mathrm{K}_{1, \mathrm{n}}$, double star $\mathrm{K}_{1, \mathrm{n}, \mathrm{n}}$, bistar $\mathrm{B}_{\mathrm{m}, \mathrm{n}}, \mathrm{Y}_{\mathrm{n}+1}, \mathrm{Tg}_{\mathrm{n}}$ and Coconut tree $\mathrm{T}(\mathrm{m}, \mathrm{n})$.

\section{REFERENCES}

1. D.M. Burton, Elementary number theory, Brown Publishers, Second edition (1990).

2. J. Devaraj, S.P.Reshma and C.Sunitha, On triangular sum graphs of some graphs, International Journal of Advanced and Innovative Research, 6 (2017) 8.

3. F.Harrary, Graph theory, Narosa publishing House (2001).

4. J.A.Gallioan, A dynamic survey of graph labeling, The Electronics Journal of Combinatorics, 17 (2010) \# DS6.

5. S.Murugesan, D.Jayaraman and J.Shiama, Second and third order triangular sum labeling of graphs, Intern. Journal of Mathematical Archive, 4(2) (2013) 55-62.

6. M.A.Seoud and M.A Salim, Further results on triangular sum graphs, International Mathematical Form, 7(48) (2012) 2393-2405.

7. P.Shankaran, On triangular sum labelings of graphs, Global Research and Development Journal for Engineering, 3 (2018) 29-33.

8. S.K.Vaidya and N.H.Shah, Some star and bistar related divisor cordial graphs, Annals of Pure and Applied Mathematics, 3(1) (2013) 67-77.

9. S.K.Vaidya, U.M.Prajapatti and P.L.Vihol, Some important results on triangular sum graphs, Applied Mathematical Sciences, 3(36) (2009) 1763-1772.

10. D.Vijayalakshmi and S.Priyanka, Star-in-coloring of some star graph families, Annals of Pure and Applied Mathematics, 14(3) (2017) 461-464. 